\title{
A pharmacist-led medication switch protocol in an academic HIV clinic: patient knowledge and satisfaction
}

Sarah S. Lee' ${ }^{1}$, Joshua P. Havens², Harlan R. Sayles³, Jennifer L. O’Neill², Anthony T. Podany ${ }^{4}$, Susan Swindells ${ }^{2}$, Kimberly K. Scarsi ${ }^{1,4}$ and Sara H. Bares ${ }^{2^{*}}$ (D)

\begin{abstract}
Background: Tenofovir alafenamide (TAF) is associated with less renal and bone toxicity compared with tenofovir disoproxil (TDF). TAF's recent FDA approval has spurred HIV providers to consider switching antiretroviral therapy (ART) regimens containing TDF to TAF to minimize long term risks. Patient views on the process of such medication switches have not been explored.

Methods: Patients taking elvitegravir/cobicistat/emtricitabine/tenofovir disoproxil fumarate (E/C/F/TDF) following the Food and Drug Administration's (FDA) approval of elvitegravir/cobicistat/emtricitabine/tenofovir alafenamide (E/C/F/TAF) received medication education from an HIV pharmacist prior to switching to the tenofovir alafenamide (TAF) formulation. Patients were asked to complete a cross-sectional survey assessing satisfaction with the switch process and knowledge about the new medication 4 to 8 weeks post-switch.

Results: Sixty five patients completed the switch and 57 (88\%) completed a follow-up survey. Most (86\%) reported understanding why the switch was made, while $91 \%$ correctly identified that TAF is associated with reduced renal toxicity, and $73 \%$ correctly identified that TAF is associated with reduced bone toxicity. No statistically significant difference was found in satisfaction with or understanding of why the medication switch was made when assessed by sex, age, race, or education, but there was a trend toward significance in the distribution of answers based on education level with those with a high school diploma, General Educational Development (GED) or less being more likely to be satisfied with the medication switch ( $p=0.074)$.
\end{abstract}

Conclusions: Education from an ambulatory clinic-based HIV pharmacist resulted in high rates of patient satisfaction and understanding of the switch from TDF to TAF-containing ART.

Keywords: HIV infection, Medication switch, Survey, Pharmacist, Patient knowledge and attitudes

\section{Background}

Currently available antiretroviral therapy (ART) regimens have led to marked declines in HIV-related morbidity and mortality [1-3]. As a result, clinical attention is increasingly focused on choosing ART regimens that optimize tolerability, long-term safety, and efficacy.

Elvitegravir/cobicistat/emtricitabine/tenofovir disoproxil fumarate $(\mathrm{E} / \mathrm{C} / \mathrm{F} / \mathrm{TDF})$ is a widely-used fixed dose combination tablet recommended as first-line therapy for

\footnotetext{
* Correspondence: sara.bares@unmc.edu

${ }^{2}$ Section of Infectious Diseases, University of Nebraska Medical Center,

Omaha, NE, USA

Full list of author information is available at the end of the article
}

treatment-naïve HIV-1 infected adults and adolescents [1]. Although $\mathrm{E} / \mathrm{C} / \mathrm{F} / \mathrm{TDF}$ has a favorable safety and tolerability profile, the TDF component carries risk of nephrotoxicity and has been shown to result in a greater decline in bone mineral density (BMD) than abacavir, the other recommend nucleoside transcriptase inhibitor $[1,4,5]$.

In November 2015, the U.S. Food and Drug Administration (FDA) approved elvitegravir/cobicistat/emtricitabine/ tenofovir alafenamide (E/C/F/TAF) for both HIV treatment naïve and experienced patients. TAF is a pro-drug of tenofovir that achieves higher intracellular concentrations of its active metabolite, tenofovir diphosphate, and results in lower plasma tenofovir concentrations compared with

(c) The Author(s). 2018 Open Access This article is distributed under the terms of the Creative Commons Attribution 4.0 International License (http://creativecommons.org/licenses/by/4.0/), which permits unrestricted use, distribution, and reproduction in any medium, provided you give appropriate credit to the original author(s) and the source, provide a link to the Creative Commons license, and indicate if changes were made. The Creative Commons Public Domain Dedication waiver (http://creativecommons.org/publicdomain/zero/1.0/) applies to the data made available in this article, unless otherwise stated. 
tenofovir disoproxil fumarate (TDF). Switching from TDF to TAF is associated with an improvement in proteinuria and renal biomarkers and smaller declines in bone mineral density [5]. This is particularly advantageous in people with underlying bone and kidney disease or those at high risk for these conditions. Importantly, these benefits have been seen without any compromise in virologic efficacy $[1,6-8]$.

While some benefits for switching from TDF to TAFbased ART regimens have been demonstrated, the ideal method for making the switch is less clear. HIV specialized pharmacists are increasingly involved in patient care, providing a significant amount of the medication teaching in HIV clinics across the United States and monitoring adherence with interventions when indicated [9]. Community pharmacy and clinic pharmacist-led HIV medication therapy management programs have resulted in improvements in medication adherence and reductions in medication errors [10-12]. Having pharmacists initiate predetermined medication switches provides efficiency for the health care team. The majority of respondents in a study exploring patient views of a pharmacist-led statin switch process were satisfied with the switch and indicated a preference for face-to-face consultation regarding the switch [13]. We are not aware of any data regarding preferred switch methods in the HIV literature.

In anticipation of switching patients from $E / C / F / T D F$ to $\mathrm{E} / \mathrm{C} / \mathrm{F} / \mathrm{TAF}$, we designed a pharmacist-led switch protocol and survey to assess patient knowledge and opinions regarding the switch. We hypothesized that the pharmacist-led switch protocol would result in high levels of patient knowledge and satisfaction.

\section{Methods}

The purpose of this research was to implement the switch from $E / C / F / T D F$ to $E / C / F / T A F$ using clinic-based pharmacists. The primary objective of the study was to assess patient satisfaction with the method in which the medication switch from E/C/F/TDF to E/C/F/TAF was made. Patient knowledge of the reason(s) why the medication switch was made was also assessed.

\section{Participant characteristics}

Inclusion criteria for the study were that potential participants were attending the University of Nebraska Medical Center (UNMC) HIV clinic, were adults who speak English, were currently receiving E/C/F/TDF and were willing and able to provide informed consent. Those with HIV RNA level more than 50 copies $/ \mathrm{mL}$ after at least 6 months on antiretroviral therapy, or receiving concomitant therapy contraindicated with $\mathrm{E} / \mathrm{C} /$ F/TAF were excluded [14].

\section{Study procedures}

Potentially eligible patients were either approached by the clinical pharmacist at the time of their clinic appointment or contacted by telephone to discuss the switch from E/C/F/TDF to E/C/F/TAF. Patients who were contacted by phone were given the option of either coming to the clinic for a face-to-face meeting with the clinical pharmacist or receiving information regarding the medication switch over the phone. As part of the pharmacist-led switch protocol, the clinical pharmacist assessed drug-drug interactions, provided medication and adherence counseling, prescribed the new medication under a collaborative drug therapy management (CDTM) agreement, and updated the medical record. Visits with the clinical pharmacist took place independently of visits with physicians and advanced practice providers. A brief written handout containing information about $\mathrm{E} / \mathrm{C} / \mathrm{F} / \mathrm{TAF}$ and outlining the benefits it provides over $\mathrm{E} / \mathrm{C} / \mathrm{F} / \mathrm{TDF}$ was offered to each participant. Following the medication switch, participants were invited to complete a study survey (see Additional file 1). The survey, designed to assess their knowledge and satisfaction with the switch process, was completed either in person or by telephone 4 to 8 weeks after the medication switch was made.

\section{Survey instrument}

An 18-item survey consisting of closed-ended questions was designed by the investigators and modeled after a similar study of patient views regarding implementation of a statin medication switch [13]. The purpose of the survey was to assess patient knowledge and opinions regarding the medication switch. Demographic domains included gender, age, education level, insurance, and payer for antiretroviral therapy. Satisfaction and knowledge questions regarding the medication switch were assessed using a five-point Likert scale of agreement/ disagreement.

\section{Statistical analysis}

Basic descriptive statistics (frequencies and percentages) were calculated for all survey questions. Demographic characteristics of respondents were examined for the entire sample while the primary outcomes of interest, including satisfaction with the method in which the medication switch was made and knowledge regarding why the switch was made, were examined both overall and by demographic subgroups within the sample. Exact Pearson chi-square tests were used to assess the associations between satisfaction, knowledge, and all demographic variables except for education level, for which an exact Mantel-Haenszel chi-square test was used. $P$-values less than 0.05 were considered significant. 


\section{Results}

\section{Participant demographic data}

A total of 65 patients underwent the medication switch from $\mathrm{E} / \mathrm{C} / \mathrm{F} / \mathrm{TDF}$ to $\mathrm{E} / \mathrm{C} / \mathrm{F} / \mathrm{TAF}$, and 57 (88\%) completed the follow-up survey between March 16, 2016 and August 16, 2016. The demographic characteristics of the respondents are outlined in Table 1 . The majority of respondents were male (89\%), white $(60 \%)$, and at least 40 years of age (53\%). Most (82\%) reported some college education or higher. Greater than half $(63 \%)$ were privately insured, while $24 \%$ were uninsured and received medications from the Nebraska AIDS Drug Assistance Program. Most $(44,77 \%)$ participants reported being informed of the switch in person, 5 (9\%) of the participants reported being informed of the switch over the telephone, and 7 (12\%) reported being informed of the switch both by telephone and in person.

\section{Satisfaction and knowledge regarding switch}

Overall, 54 (96\%) of the participants agreed or strongly agreed that they were satisfied with how the switch was explained to them. Most (86\%) reported understanding why the switch was made, $91 \%$ correctly identified that TAF is associated with reduced renal adverse effects, and $73 \%$ correctly identified that TAF is associated with

Table 1 Characteristics of survey respondents $(n=57)$

\begin{tabular}{ll}
\hline & $\mathrm{n}(\%)$ \\
\hline Sex & $51(89)$ \\
Female & $6(11)$ \\
Age Group & \\
$<40$ years & $27(47)$ \\
$\geq 40$ years & $30(53)$ \\
Race & \\
Non-Hispanic White & $35(61)$ \\
Non-Hispanic Black & $13(23)$ \\
aOther & $9(16)$ \\
Education & \\
HS diploma or GED or below & $11(19)$ \\
Some college or 2 year associate's degree & $33(58)$ \\
Bachelor's degree or greater & $13(23)$ \\
Health Insurance & \\
Private & $23(40)$ \\
Uninsured & $14(24)$ \\
ADAP-sponsored health insurance & $13(23)$ \\
Medicaid/Medicare & $5(9)$ \\
Unknown & $2(4)$ \\
\hline
\end{tabular}

${ }^{\mathrm{a} O t h e r}$ includes Asian/Pacific Islander, White and Native American, White and Hispanic, and Black and Native American, as well as those reporting "Other" HS high school, GED general education diploma
Table 2 Satisfaction and knowledge following the medication switch

\begin{tabular}{ll}
\hline & $\begin{array}{l}\text { Strongly Agree } \\
\text { or Agree n (\%) }\end{array}$ \\
\hline I am happy that my Stribild was switched to Genvoya. & $40(70)$ \\
I am happy with the way in which the switch was & $54(96)$ \\
explained to me. & \\
I do not understand why my Stribild has been switched. & $8(14)$ \\
My medication was switched because Genvoya is & $51(91)$ \\
better for my kidneys than Stribild. & $41(73)$ \\
$\begin{array}{l}\text { My medication was switched because Genvoya is } \\
\text { better for my bones than Stribild. }\end{array}$ & $47(82)$ \\
Genvoya and Stribild are both equally effective in \\
treating HIV.
\end{tabular}

reduced bone adverse effects, as outlined in Table 2 . In regard to the brief handout that was given to all patients, only 40 (70\%) respondents reported receiving written information about the new medication. Of those, 33 (83\%) said that the written information was helpful.

\section{Relationship between demographic variables and knowledge and satisfaction}

There was no statistically significant difference in satisfaction with or understanding of why the medication switch was made when assessed by sex, age, race, or education, but there was a trend toward significance in the distribution of answers based on education level, with those with a high school diploma, General Educational Development (GED) or less being more likely to be satisfied with the medication switch $(p=0.074)$. Respondents receiving Medicaid or Medicare were statistically less likely to understand why their $\mathrm{E} / \mathrm{C} / \mathrm{F} / \mathrm{TDF}$ had been switched $(p=0.037)$ compared to survey respondents with other forms of health insurance.

\section{Discussion}

Our study evaluated patient satisfaction and knowledge following a pharmacist-led medication switch protocol. Overall, patient satisfaction with the switch process was high. Similarly, patient-reported and objective knowledge levels about the reason for the switch were high. This demonstrates the successful introduction of a pharmacist-led medication switch strategy in an HIV clinic. We aim to use the results to inform the implementation of future medication switch protocols.

While age, gender, race and education were not independently associated with knowledge and/or satisfaction with the medication switch, there was a trend toward significance in the distribution of answers based on education level, with those having less education being more likely to be satisfied with the medication switch. Although those receiving Medicaid/Medicare were less likely to understand the reason for their medication 
switch, the small number $(n=5)$ reporting this form of insurance limits the interpretation of these results.

Patients were given the option of receiving medication teaching by telephone, but most (89\%) opted to receive the information in person, or over the telephone in addition to an in-person consultation. This is consistent with the results of other medication switch studies [13].

There were several limitations to this study. The survey was conducted at a single site and included a small number of participants as well as few women and young (age $<27$ years old) participants, which limits the overall generalizability of the results. Additionally, while it would have been preferable for all respondents to complete the survey either in person or by phone, we offered both options in order to increase the response rate. This may have resulted in variability in the understanding or interpretation of survey questions as well as reporting of results based on the method of survey completion.

\section{Conclusions}

We conclude that patient education from an ambulatory clinic-based HIV specialist pharmacist resulted in high rates of patient satisfaction and understanding of the switch from TDF to TAF-containing ART. The high rates of satisfaction and knowledge in this pharmacist-led medication switch protocol suggest that pharmacists could lead similar medication switches in the future yielding similar results, and face-to-face consultation should be considered given patient preference for this mode.

\section{Additional file}

Additional file 1: Patient Survey. 18-item survey tool designed to assess patient knowledge and satisfaction regarding the switch from E/C/F/TDF to E/C/F/TAF. (PDF $23 \mathrm{~kb}$ )

\section{Abbreviations}

ART: Antiretroviral therapy; CDTM: Collaborative drug therapy management; E/C/F/TAF: Elvitegravir/cobicistat/emtricitabine/tenofovir alafenamide; E/C/F/ TDF: Elvitegravir/cobicistat/emtricitabine/tenofovir disoproxil fumarate; FDA: Food and Drug Administration; GED: General Educational Development; TAF: Tenofovir alafenamide; TDF: Tenofovir disoproxil fumarate; UNMC: University of Nebraska Medical Center

\section{Acknowledgements}

We thank all the respondents for their participation in this study, and Deanna Hansen for outstanding administrative support.

\section{Funding}

This study was funded by Gilead Sciences, Inc. through an investigator-initiated grant to the corresponding author (SHB).

\section{Availability of data and materials}

The datasets used and/or analyzed during the current study are available from the corresponding author on reasonable request.

\section{Authors' contributions}

SHB, SSL, JPH, ATP, SS and KKS designed the study and wrote the protocol. HRS was responsible for statistical analyses and technical input for the survey content. JLO coordinated the participant's visits and study procedures. All authors read and approved the final manuscript.

Ethics approval and consent to participate

The study was approved by the Institutional Review Board of the University of Nebraska Medical Center, IRB number 637-15. Exemption from written informed consent was granted and a cover letter informing participants about the study was attached to each survey.

\section{Consent for publication}

$\mathrm{N} / \mathrm{A}$

\section{Competing interests}

SHB reports research grants to her institution from Gilead Sciences, for this work. SS reports research grants to her institution from ViiV and Merck. The other authors declare that they have no competing interests.

\section{Publisher's Note}

Springer Nature remains neutral with regard to jurisdictional claims in published maps and institutional affiliations.

\section{Author details}

${ }^{1}$ College of Medicine, University of Nebraska Medical Center, NE, Omaha, NE 68198-8106, USA. ${ }^{2}$ Section of Infectious Diseases, University of Nebraska Medical Center, Omaha, NE, USA. ${ }^{3}$ College of Public Health, University of Nebraska Medical Center, Omaha, NE, USA. ${ }^{4}$ College of Pharmacy, University of Nebraska Medical Center, Omaha, NE, USA.

Received: 20 December 2017 Accepted: 29 June 2018

Published online: 06 July 2018

\section{References}

1. Panel on Antiretroviral Guidelines for Adults and Adolescents. Guidelines for the use of antiretroviral agents in HIV-1 infected adults and adolescents. Department of Health and Human Services. Available at http://aidsinfo.nih. gov/ContentFiles/AdultandAdolescentGL.pdf.

2. Guaraldi G, Orlando G, Zona S, et al. Premature age-related comorbidities among HIV-infected persons compared with the general population. Clin Infect Dis. 2011:53(11):1120-6.

3. Palella FJ Jr, Delaney KM, Moorman AC, et al. Declining morbidity and mortality among patients with advanced human immunodeficiency virus infection. HIV Outpatient Study Investigators. N Engl J Med. 1998;338(13):853-60.

4. Post FA. Managing chronic kidney disease in the older adults living with HIV. Curr Opin Infect Dis. 2017:30(1):4-11.

5. Wang H, Lu X, Yang $X, X u N$. The efficacy and safety of tenofovir alafenamide versus tenofovir disoproxil fumarate in antiretroviral regimens for HIV-1 therapy: meta-analysis. Medicine. 2016;95(41):e5146.

6. Gallant JE, Daar ES, Raffi F, et al. Efficacy and safety of tenofovir alafenamide versus tenofovir disoproxil fumarate given as fixed-dose combinations containing emtricitabine as backbones for treatment of HIV-1 infection in virologically suppressed adults: a randomised, double-blind, activecontrolled phase 3 trial. Lancet HIV. 2016;3(4):e158-65.

7. Sax PE, Wohl D, Yin MT, et al. Tenofovir alafenamide versus tenofovir disoproxil fumarate, coformulated with elvitegravir, cobicistat, and emtricitabine, for initial treatment of HIV-1 infection: two randomised double-blind, phase 3, non-inferiority trials. Lancet. 2015;

8. Wohl D, Oka S, Clumeck N, et al. Brief report: a randomized, double-blind comparison of Tenofovir Alafenamide versus Tenofovir Disoproxil fumarate, each Coformulated with Elvitegravir, Cobicistat, and Emtricitabine for initial HIV-1 treatment: week 96 results. J Acquir Immune Defic Syndr. 2016;72(1):58-64.

9. Schafer JJ, Gill TK, Sherman EM, McNicholl IR. ASHP guidelines on pharmacist involvement in HIV care. Am J Health Syst Pharm. 2016;73(7):468-94.

10. Dilworth TJ, Klein PW, Mercier RC, Borrego ME, Jakeman B, Pinkerton SD. Clinical and economic effects of a pharmacist-administered antiretroviral therapy adherence Clinic for Patients Living with HIV. J Manag Care Spec Pharm. 2018:24(2):165-72.

11. Hirsch JD, Gonzales M, Rosenquist A, Miller TA, Gilmer TP, Best BM. Antiretroviral therapy adherence, medication use, and health care costs during 3 years of a community pharmacy medication therapy management program for Medi-Cal beneficiaries with HIV/AIDS. J Manag Care Pharm. $2011 ; 17(3): 213-23$ 
12. McNicholl IR, Gandhi M, Hare CB, Greene M, Pierluissi E. A pharmacistled program to evaluate and reduce polypharmacy and potentially inappropriate prescribing in older HIV-positive patients. Pharmacotherapy. 2017:37(12):1498-506.

13. Krska J, Allison K, Delargy M, Murray L, Smith H. Implementing a statin switching programme in primary care: patients' views and experiences. Br J Clin Pharmacol. 2012;74(1):147-53.

14. Genvoya (elvitegravir, cobicistat, emtricitabine, and tenofovir alafenamide) [package insert]. Gilead Sciences, Inc. Foster City, CA; 2017. http://www. amamanualofstyle.com/view/10.1093/jama/9780195176339.001.0001/med9780195176339-div2-73.

Ready to submit your research? Choose BMC and benefit from:

- fast, convenient online submission

- thorough peer review by experienced researchers in your field

- rapid publication on acceptance

- support for research data, including large and complex data types

- gold Open Access which fosters wider collaboration and increased citations

- maximum visibility for your research: over $100 \mathrm{M}$ website views per year 